\title{
Anterior Communicating Artery Aneurysm associated with Bilateral Occlusion of the Middle Cerebral Artery -Case Report-
}

\author{
Mutsuo Kusunose, Keiji Koshu, Shigeru HiROTA, Makoto SONOBE \\ and Shinichiro TAKAHASHI
}

Department of Neurosurgery, Mito National Hospital, Mito, Ibaraki

\begin{abstract}
A female who had had no previous cerebrovascular events suffered a subarachnoid hemorrhage and was referred to our hospital. Angiography revealed an anterior communicating artery aneurysm and bilateral occlusion of the middle cerebral artery at its origin. She exhibited mild ischemic symptoms after clipping of the aneurysm, possibly as a consequence of disturbance of the collateral blood flow during surgery. This is the third such case reported in the literature.
\end{abstract}

Key words: cerebral aneurysm, middle cerebral artery, cerebrovascular occlusion, subarachnoid hemorrhage, controlled hypotension, brain retraction

\section{Introduction}

Cerebral aneurysms associated with bilateral occlusion of the middle cerebral artery (MCA) are very rare; only two such cases have been reported., ${ }^{2,3)} \mathrm{We}$ describe a third case, that of a female who presented with subarachnoid hemorrhage (SAH) and showed good recovery after clipping of the aneurysm.

\section{Case Report}

A 43-year-old female was referred to our hospital with complaints of severe headache and nausea. She had been hypertensive about 10 years but was otherwise healthy. On admission she was alert, with neck stiffness and slight right hemiparesis. Her blood pressure was $200 / 90 \mathrm{mmHg}$. Computed tomography (CT) revealed typical SAH.

Left carotid angiograms showed stenosis at the $\mathrm{C}_{2}$ portion of the internal carotid artery and complete occlusion of the left MCA. There was a saccular anterior communicating artery aneurysm at the $A_{1}$ $A_{2}$ junction of the left anterior cerebral artery (Fig. 1). On right carotid angiograms, the MCA was seen to be occluded at its origin (Fig. 2). Abundant

Received September 6, 1988; Accepted December 23, 1988 collateral circulation (leptomeningeal anastomosis) from the anterior and posterior cerebral arteries provided a retrograde supply to the bilateral MCA territories.

She underwent surgery on the day of admission, with a Hunt and Kosnik grade of 3 . We chose the "interhemispheric approach." She was anesthetized using enflurane, and $500 \mathrm{ml}$ of $20 \%$ mannitol was administered at the start of the craniotomy. In a 4-hour

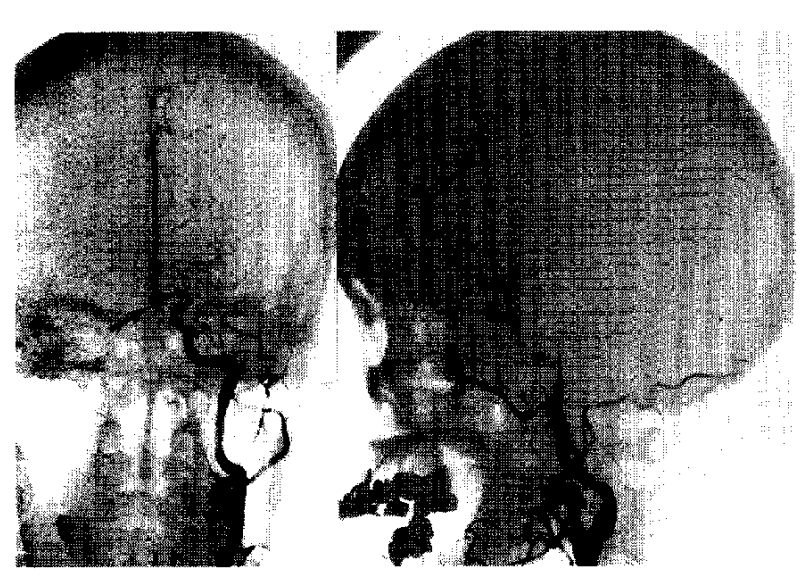

Fig. 1 Left carotid angiograms showing occlusion of the MCA. The anterior communicating artery aneurysm is also visible (arrow). 


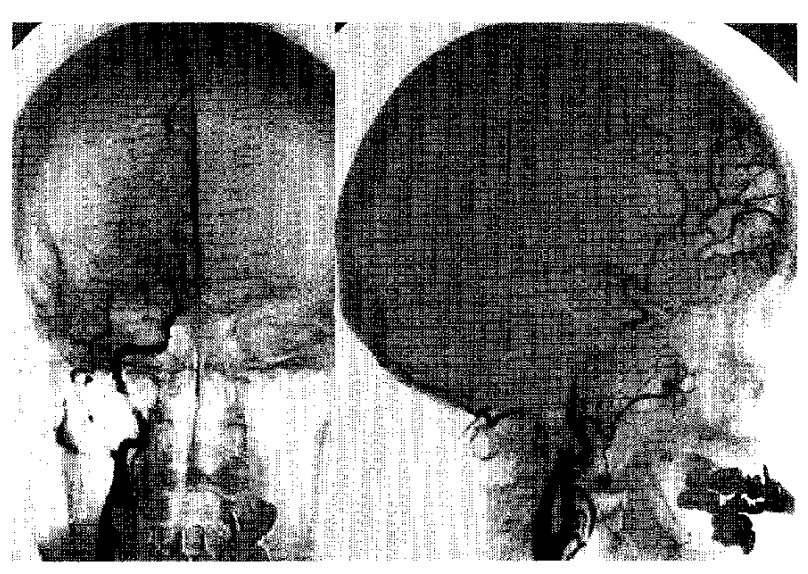

Fig. 2 Right carotid angiograms demonstrating occlusion of the MCA.

procedure, the aneurysm was clipped uneventfully, without the use of temporary clips. Her systolic blood pressure was maintained at under $130 \mathrm{mmHg}$ from the time of admission and at $100-120 \mathrm{mmHg}$ during surgery.

Postoperatively, she exhibited slight expressive aphasia and deterioration of the right hemiparesis, although she was alert. A CT scan taken on the day after surgery showed an abnormal area of mixed density in the left anterior cerebral artery territory, indicating brain damage (Fig. 3). The hemiparesis and expressive aphasia, however, improved gradually thereafter and had almost disappeared by the 11th postoperative day. Symptoms of vasospasm did not occur. A CT scan obtained on the 16th day after surgery demonstrated a small low-density area in the left anterior cerebral artery territory.

\section{Discussion}

There have been only two reported cases of cerebral aneurysm associated with bilateral occlusion of the MCA. $^{2,3)}$ Both patients were Japanese and both presented with SAH due to ruptured anterior communicating artery aneurysms.

The first case $^{3)}$ was a 62-year-old female. Her status was poor on admission and deteriorated gradually due to vasospasm, which was demonstrated angiographically on the 11th hospital day. The aneurysm was not clipped; in retrospect, the authors concluded that STA-MCA anastomosis should have been attempted in the early stage (within 3 days) to prevent cerebral ischemia, together with clipping of the aneurysm.

The second case, ${ }^{2)}$ a 60 -year-old female, had von Recklinghausen's disease. She underwent surgery on the 2nd day of hospitalization but died of complications 1 month later.

The first patient had experienced transient numbness in the left fingers and lips 3 years before the $\mathrm{SAH}$. This symptom might have been related to the MCA occlusion. On the other hand, neither the second patient nor ours had experienced any neurological symptoms prior to $\mathrm{SAH}$, and all three were neurologically normal before SAH occurred. In all three cases, angiograms disclosed abundant collateral circulation. If these three cases are representative, the prognosis for bilateral MCA occlusion alone appears good.

Bogousslavsky et al. ${ }^{1)}$ found nine cases of bilateral occlusion of the MCA trunk among 1377 patients in an international randomized trial of EC/IC Bypass Study. Seven of these patients were Japanese or Chinese (five males and two females). The authors

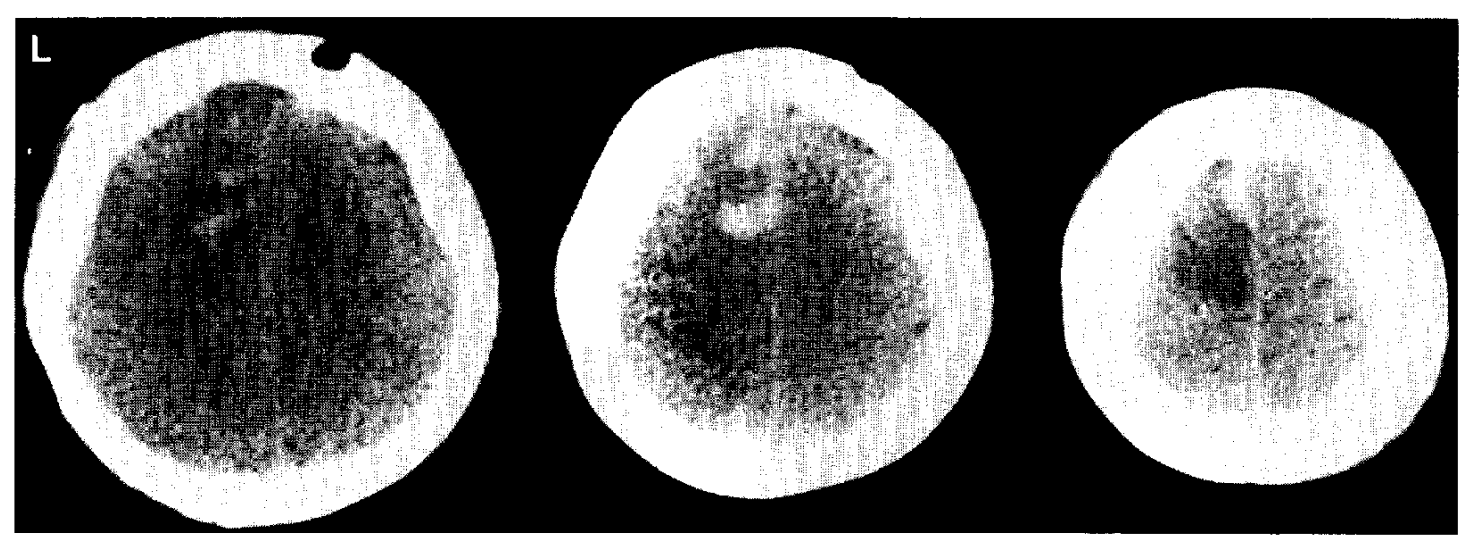

Fig. 3 A CT scan taken on the day after surgery. An area of mixed density, indicating brain damage, is seen in the left anterior cerebral artery territory. 
concluded that bilateral MCA occlusion has a good prognosis if abundant collateral circulation is present. Our patient had slight right hemiparesis after the $\mathrm{SAH}$, and stenosis was observed in the intracranial portion of left internal carotid artery. This suggests that the post-SAH collateral blood supply in the left cerebral hemisphere may not have been sufficient.

Postoperative CT indicated that some brain damage had occurred in the left anterior cerebral artery territory of our patient, possibly as a result of our maintaining her in a state of controlled hypotension after admission. In light of her history of hypertension, which perhaps was necessary to sustain sufficient collateral circulation, it may have been better to maintain a higher blood pressure. An additional contributory factor in this patient's brain damage might have been brain retraction during surgery via the interhemispheric approach. The left hemisphere may have been relatively ischemic due to the $\mathrm{SAH}$, so that even slight brain retraction produced brain damage. However, the brain damage was not severe and the patient showed fairly good recovery.

We conclude that patients with bilateral occlusion of the MCA have a good prognosis in terms of brain ischemia, provided there is abundant collateral circulation. Therefore, in patients with SAH due to aneurysm rupture, we feel that early clipping of the aneurysm can be performed without bypass surgery.
However, we would like to emphasize that blood pressure should be maintained at a level high enough to prevent brain ischemia, both before and during surgery, which can occur if the collateral circulation is compromised. Finally, brain retraction must be undertaken with great care.

\section{References}

1) Bogousslavsky J, Wong W, Barnett HJM, Fox AJ: Bilateral occlusion of the trunk of the middle cerebral artery. Results of an international randomized trial. The EC/IC Bypass Study Group. Stroke 17: 11071111,1986

2) Chono $Y$, Ueno $K$, Nunomura $M$, Ohta $Y$, Nomura $M$, Nakamura N: Von Recklinghausen's disease associated with occlusion of bilateral middle cerebral artery, moyamoya phenomenon, and an anterior communicating artery aneurysm. Report of an autopsy case. Neurol Med Chir (Tokyo) 25: 209-214, 1985 (in Japanese)

3) Tazawa $T$, Mizukami $M$, Togashi $O$, Hyodo $A$, Eguchi $\mathrm{T}$ : Anterior communicating artery aneurysm with bilateral middle cerebral artery occlusion. Case report. Neurol Med Chir (Tokyo) 23: 478-482, 1983 (in Japanese)

Address reprint requests to: S. Takahashi, M.D., Department of Neurosurgery, Mito National Hospital, 3-21 Higashihara, Mito, Ibaraki 310, Japan. 